\title{
Medulloblastoma, Genetically Defined
}

National Cancer Institute

\section{Source}

National Cancer Institute. Medulloblastoma, Genetically Defined. NCI Thesaurus. Code C129439.

A term that refers to the classification of medulloblastomas according to their molecular characteristics. 